\title{
NPP VIIRS Early On-Orbit Geometric Performance
}

\author{
Robert E. Wolfe, GSFC, USA \\ Guoqing Lin, Innovim, USA \\ Masahiro Nishihama, Sigma Space, USA \\ Krishna P. Tewari, Innovim, USA \\ Enrique Montano, Sigma Space, USA
}

The NASA/NOAA Visible Infrared Imager Radiometer Suite (VIIRS) instrument on-board the Suomi National Polar-orbiting Partnership (NPP) satellite was launched in October, 2011. The instrument geometric performance includes sensor spatial response, band-to-band co-registration (BBR), and geolocation accuracy and precision. The geometric performance is an important aspect of sensor data record (SDR) calibration and validation. In this paper we will discuss geometric performance parameter characterization using the first seven-month of VIIRS' earth and lunar data, and compare with the at-launch performance using ground testing data and analysis of numerical modeling results as the first step in on-orbit geometric calibration and validation.

The instrument sensor (detector) spatial response is estimated by line spread functions (LSFs) in the scan and track directions. The LSFs are parameterized by dynamic field of view (DFOV) in the scan direction and instantaneous FOV (IFOV) in the track direction, modulation transfer function for the 16 moderate resolution bands (M-bands), and horizontal spatial resolution for the five imagery bands (I-bands). VIIRS BBR for M-bands and I-bands is operationally defined as the overlapped fractional area of angular pixel sizes from the corresponding detectors in a band pair, including nested I-bands into M-bands. VIIRS geolocation accuracy and precision are affected by instrument parameters, ancillary data (i.e., ephemeris and attitude), and thermally induced pointing variations with respect to orbital position. These are being tracked by a ground control point matching (CPM) program and corrected in geolocation parameter lookup tables (LUTs) in the ground data processing software. 